\title{
Evolution in Private Practice Interventional Radiology: Data Mining Trends in Procedure Volumes
}

\author{
James J. Morrison, $\mathrm{MD}, \mathrm{MBI}^{1}$ \\ ${ }^{1}$ Advanced Radiology Services, PC, Grand Rapids, Michigan \\ Semin Intervent Radiol 2019;36:17-22
}

\begin{abstract}
Address for correspondence James J. Morrison, MD, MBI, Advanced Radiology Services, PC, 3264 North Evergreen Drive, Grand Rapids, MI 49525
\end{abstract}

\author{
Abstract \\ Keywords \\ - interventional \\ radiology \\ - private practice \\ - practice pattern
}

Diversity of procedures is a mainstay of interventional radiology (IR) and the ability to adapt and acquire new procedural skills is a benefit in a constantly changing medical environment. Strategies for success include building direct referral patterns from primary care providers, fostering strong interpersonal relationships with referring services, and providing efficient care coordinated through a dedicated IR clinic. In this study, retrospective analysis of procedural volumes over 16 years within a single large private IR practice was performed to examine the evolution of private practice IR. Primary data are presented, with analysis of the internal and external factors that have determined current procedural scope.
Interventional radiology (IR) has evolved beyond angiography and "special procedures." 1 As a specialty, IR prides itself in part on the ability to solve problem, adapt, and innovate new therapies using imaging guidance. However, the scope and breadth of procedures performed varies widely across institutions, geographic regions, and individual practice patterns. ${ }^{2}$ The reasons for these variations are multifactorial and are the combined result of external forces and internal practice decisions.

External forces include hospital service agreements, credentialing, service overlap with competing specialties, and facility or equipment availability. Internal factors include competency and training of procedural physicians as well as willingness or interest in providing or expanding services. All of these factors have played a role in the evolution of our practice model.

The purpose of this retrospective analysis is to examine the evolution of our practice utilizing procedure volume data from a variety of service lines to gain a better understanding of those forces and decisions which have influenced our scope of practice over the last decade and a half.

\section{Materials and Methods}

Electronic procedural records were available from our primary hospital PACS beginning in 2002. In addition, procedural billing records were available from the partnership's accounting service beginning in 2013. Data from both of these sources were queried and used for analysis of all IR procedures performed from January 2002 through July 2018. The resultant dataset summarized the IR procedure volumes by month and year. These data were then further subanalyzed using spreadsheet software (Microsoft Excel, Redmond, WA) to yield procedure volume trends over time.

A subset of procedures was then chosen to exemplify the evolution of service lines over the study period. Service lines, as defined by their individual procedures, can be seen in $\boldsymbol{- T a b l e} \mathbf{1}$.

Normalization of procedure naming had to be performed, as the "Orderable" terms changed several times over the study period. For instance, paracentesis consisted of two orderables: "Interventional Radiology Paracentesis with Image Guidance" and "IR PARACENTESIS WITH IMAGE GUIDANCE." Many of the name changes were due to a switch in radiology information system (RIS) as the hospital system adopted a new electronic health record. In addition, a single procedure may result in creation of multiple procedure orderables. For example, treatment of a peripheral arterial stenosis could involve orderables of angiography, angioplasty, and stent placement. These were consolidated when possible. Finally, more specific procedure names have been added to the RIS over time, such as "IR EMBOLIZATION TUMOR" to describe oncologic transarterial embolization. The use of billing data introduced additional complexity in analysis, as the billing codes do not always correspond to a procedure orderable in a 1:1 relationship. Use of billing data was confined to analysis of neurointerventional (NIR)
Issue Theme A Private Practice Perspective; Guest Editor, Manish K. Varma, MD
Copyright (C) 2019 by Thieme Medical Publishers, Inc., 333 Seventh Avenue, New York, NY 10001, USA. Tel: +1(212) 584-4662.
DOI https://doi.org/ 10.1055/s-0039-1683358. ISSN 0739-9529. 
Table 1 Service lines and the procedures associated with them

\begin{tabular}{|l|l|l|l|l|}
\hline Spinal intervention & Interventional oncology & Neurointervention & Peripheral arterial disease & Minor procedures \\
\hline Vertebroplasty & SIRT & Cerebral angiography & Angiography & Paracentesis \\
Kyphoplasty & Ablation & Spinal angiography & Angioplasty & Thoracentesis \\
Sacroplasty & TACE & Aneurysm coiling & Atherectomy & Lumbar puncture \\
& DEB-TACE & Carotid stenting & Stent placement & PICC \\
& & Cerebral stenting & & \\
& Wada testing & & \\
& Thrombectomy & & \\
\end{tabular}

Abbreviations: DEB-TACE, drug-eluting bead transarterial chemoembolization; PICC, peripherally inserted central catheter; SIRT, selective internal radiation therapy; TACE, transarterial chemoembolization.

procedures and a corrected ratio of orderables to billing codes was used to estimate total procedures. In total, the derived data represent a high-level evaluation of the procedural trends over the study period.

\section{Results}

Total procedural volumes during the study period show a steady increase in IR procedures since 2003 (-Fig. 1). Data from 2002 were found to be incomplete and data from 2018 were only available through July; both of these years were subsequently excluded from the trend analysis.

Growth of interventional oncology procedures such as selective internal radiation therapy (SIRT) and ablation has been more rapid over the past decade. Ablation procedure names were added in 2005. The SIRT service line began in 2009. Transarterial tumor embolization procedures only received a dedicated procedure name in the RIS in 2014, so while the procedure was being performed prior to 2014, the procedure volumes are not easily separable from the generic “embolization" procedures (-Fig. 2). Vertebroplasty experienced a steady increase in volume after the service line was first initiated, followed by a decline before settling into a consistent volume between 150 and 200 per year over the past 5 years (- Fig. 3 ). NIR procedures were a consistent volume of yearly procedures until 2014 when the volume began to increase. The number of NIR procedures performed by our private practice physicians has decreased precipitously despite an overall increase in the institutional numbers over the past 3 years (-Fig. 4). Peripheral arterial diagnostics and intervention has exhibited a steady decline in volume throughout the study period (-Fig. 5).

Finally, minor procedures including paracentesis, thoracentesis, and lumbar punctures have grown considerably in volume over the study period (-Fig. 6). Peripherally inserted central catheter (PICCs) volumes have decreased steadily over the past decade. The initiation of a dedicated vascular access team near the end of 2017 has nearly eliminated them from our IR practice altogether ( - Fig. 7).

\section{Discussion}

One of the unique aspects of IR is the depth and breadth of minimally invasive image-guided procedures. Forces driving the scope of practice of an individual interventional radiologist include those internal to the practice and individual practitioner as well as external forces related to contractual agreements and the environment within partner health systems. All of these forces have played a role in defining our current state of service lines.

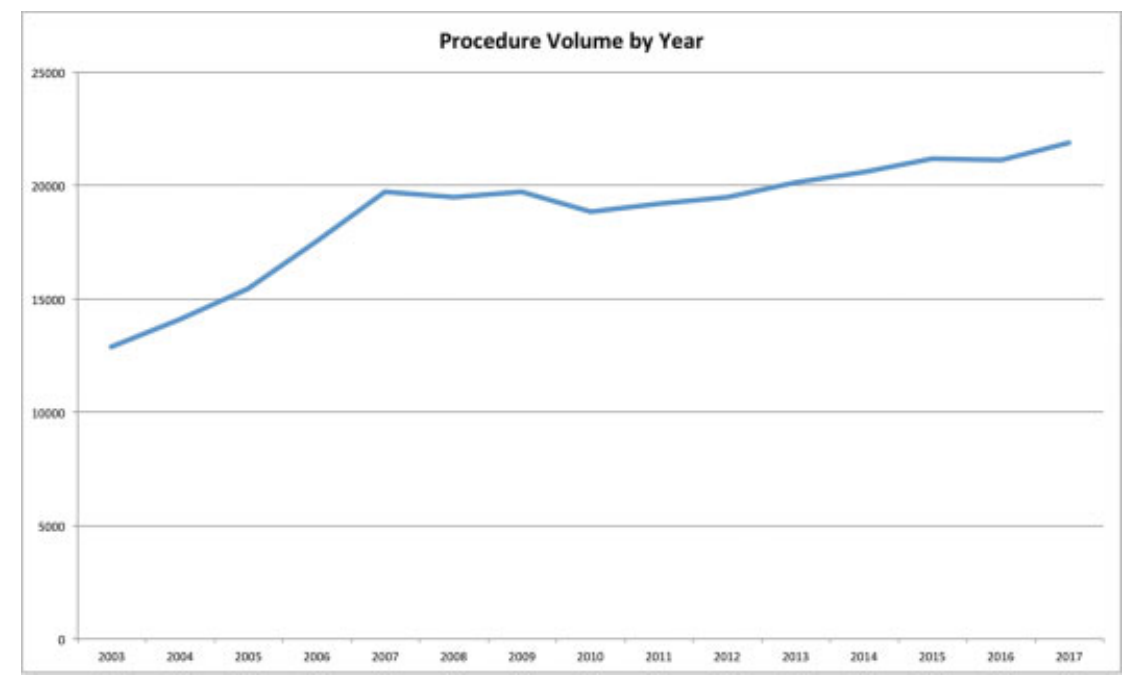

Fig. 1 Procedure volume by year. 


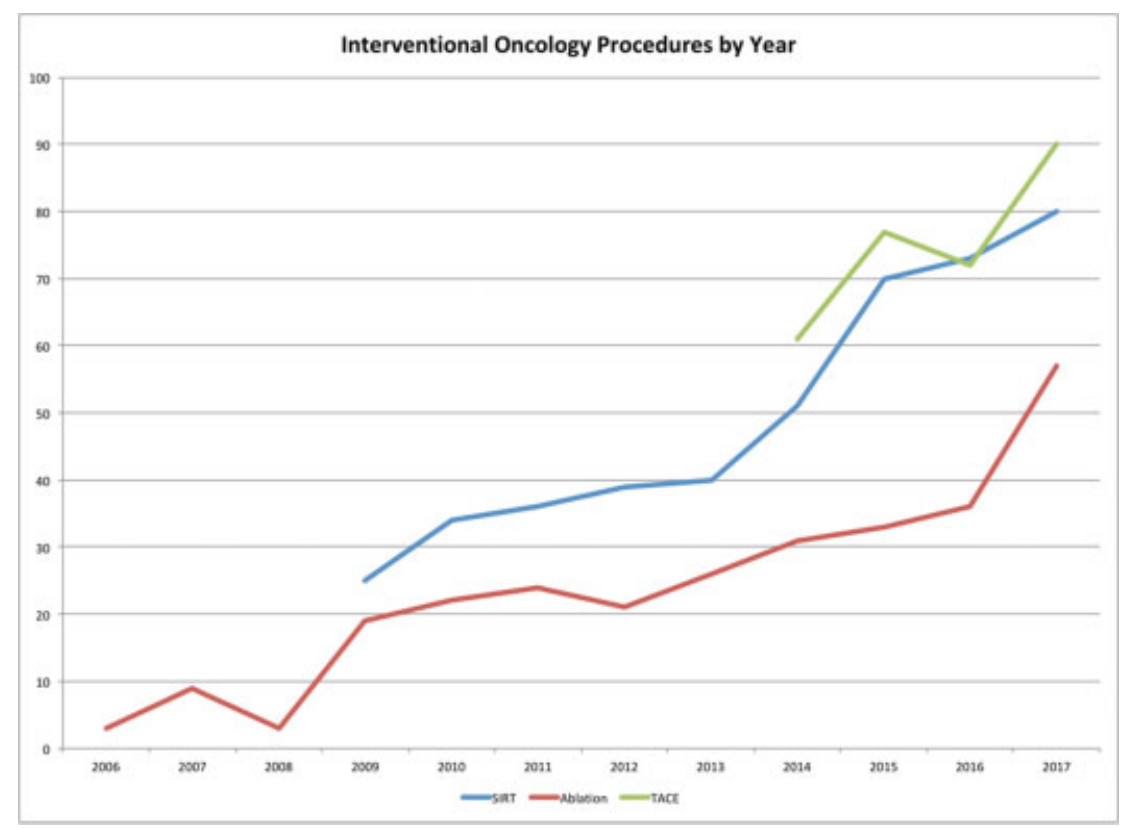

Fig. 2 Interventional oncology procedure volumes by year.

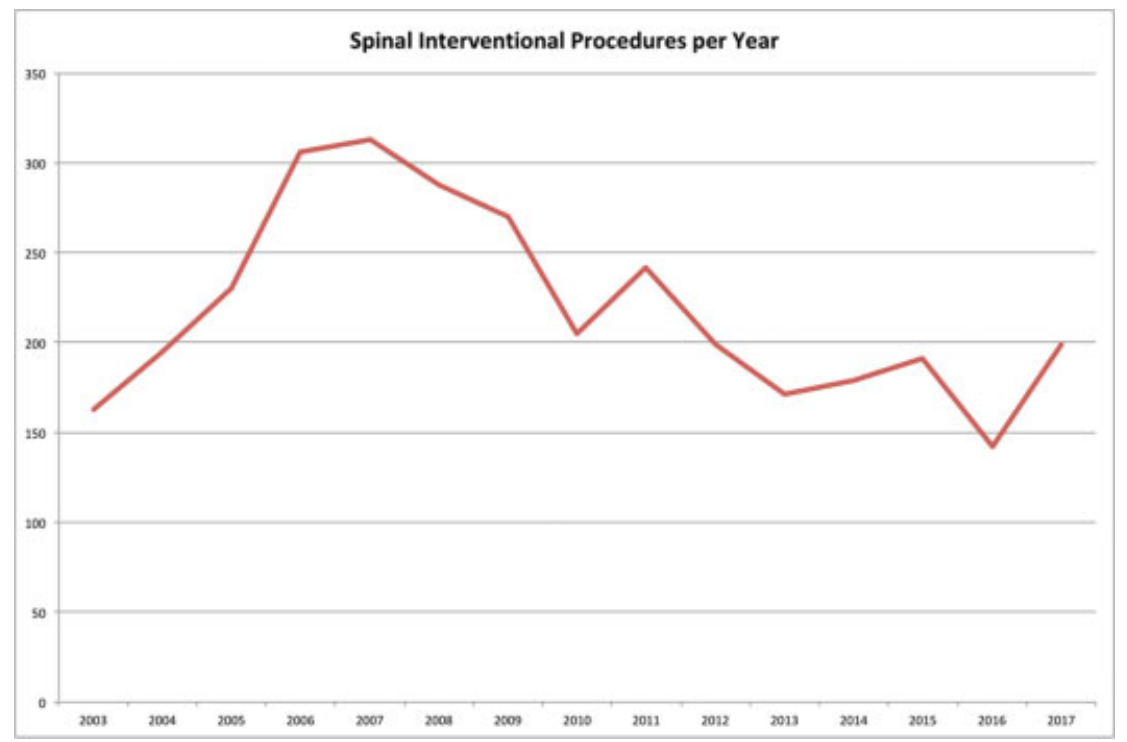

Fig. 3 Spinal intervention procedures by year.

Interventional oncology has expanded in our practice in parallel with the growth of oncologic intervention across the country. The primary drivers of our success in growing our referral base is through participation in multidisciplinary tumor conferences and developing the personal and collegial relationships with our surgical and medical oncologists. Having IRs dedicated to interventional oncology who are responsive to the needs of the surgical and medical oncologists regarding medical decision making has helped establish our service line as the fourth pillar of oncologic care. Similarly, our willingness to participate in clinical trials has helped solidify our position among our oncology colleagues.

Interventional spine procedures were started in 2002 and peaked in volume in 2007. There was a decline in volume commensurate with the publication of two sham studies in the
New England Journal of Medicine showing no benefit of vertebroplasty versus placebo., ${ }^{3,4}$ The volume has since plateaued between 150 and 200 procedures a year. We have evolved our vertebroplasty practice to follow newer data supporting the use of early intervention for acute vertebral fractures. The ability to intervene early has been buoyed by the efficiency of our outpatient clinic in moving from referral to consultation and treatment, limiting delays to achieve optimal results for our patients. Using an outpatient clinic model similar to the one described by Siskin, we have secured our role as spine interventionists in the region with a referral pattern built directly to primary care providers. ${ }^{5}$

Peripheral arterial intervention has suffered a long slow decline. This began with the loss of exclusivity for endovascular intervention during contract negotiations in the early/ 


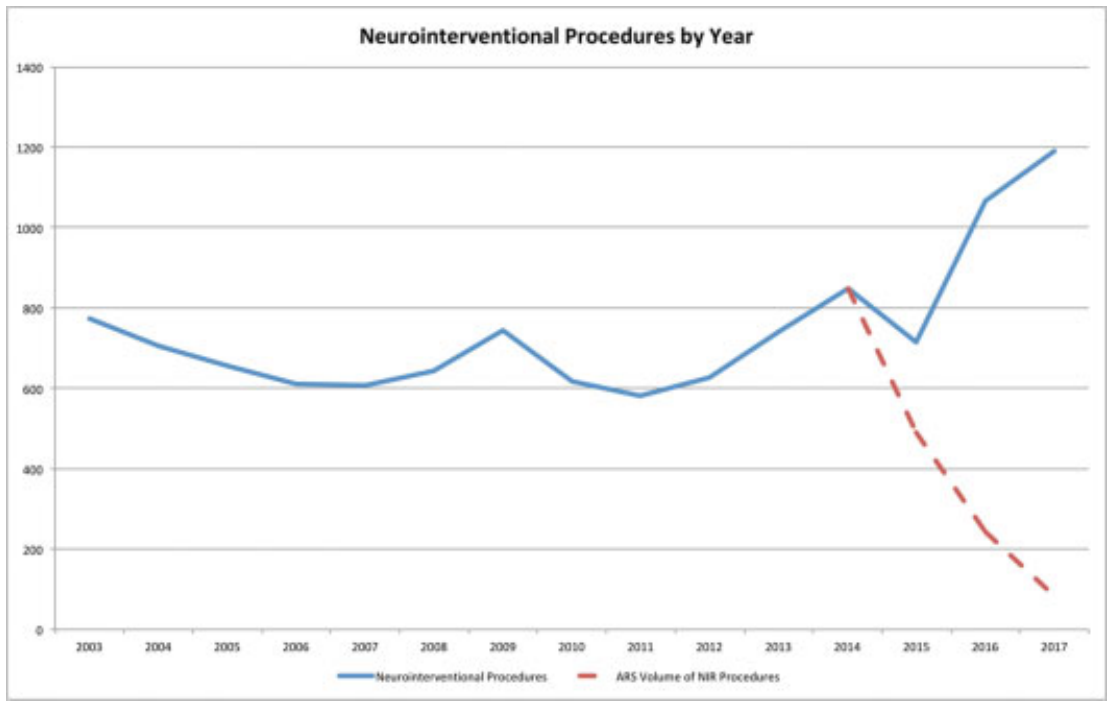

Fig. 4 Neurointerventional procedures by year.

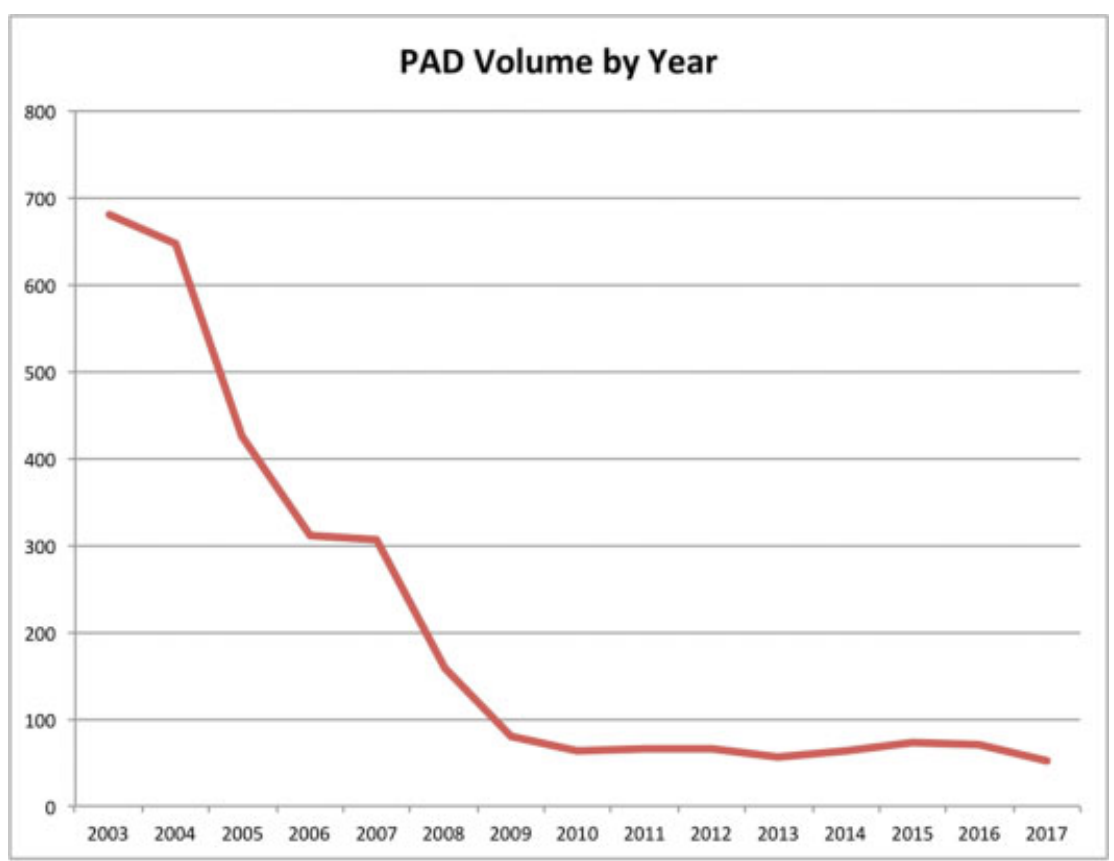

Fig. 5 Peripheral arterial disease (PAD) intervention by year.

mid-2000s, allowing vascular surgeons and cardiologists the credentialing needed to begin performing these procedures in the same hospital system. This loss of exclusivity was another battle in the "Turf War" which has played out between IR and nonradiologists throughout the country. ${ }^{6}$ The peripheral arterial services declined even further after an internal decision made by the partners to cede arterial thrombolysis cases to vascular surgery.

NIR procedures were a cornerstone of our practice until 2015 , when contract negotiations with our primary hospital system resulted in loss of exclusivity for NIR. Specifically, the hospital system pressured heavily for a monolithic approach to stroke care, opening the door to both interventional neurologists and neurosurgeons participating in NIR procedures. The decision to ultimately relinquish NIR was based primarily on the hospital's decision to have a dedicated Neurosciences Service Line directed by a highly recruited and high-profile neurologist and neurosurgeon, and our model with interventionalists who also did neurointervention did not fit. Given the organization's commitment to their new model and the leverage over us during contract negotiations, we decided that it was better for the group as a whole to cede NIR to the hospital than to fight and risk negative repercussions to our contract. Subsequently, the referral patterns for NIR procedures changed and our primary NIR left the practice. The NIR service is now performed exclusively by the hospital-employed physicians.

Throughout the past 15 years, there has been a steady increase in volume of smaller procedures, which threatens to overwhelm procedural time for larger cases. Many of these 

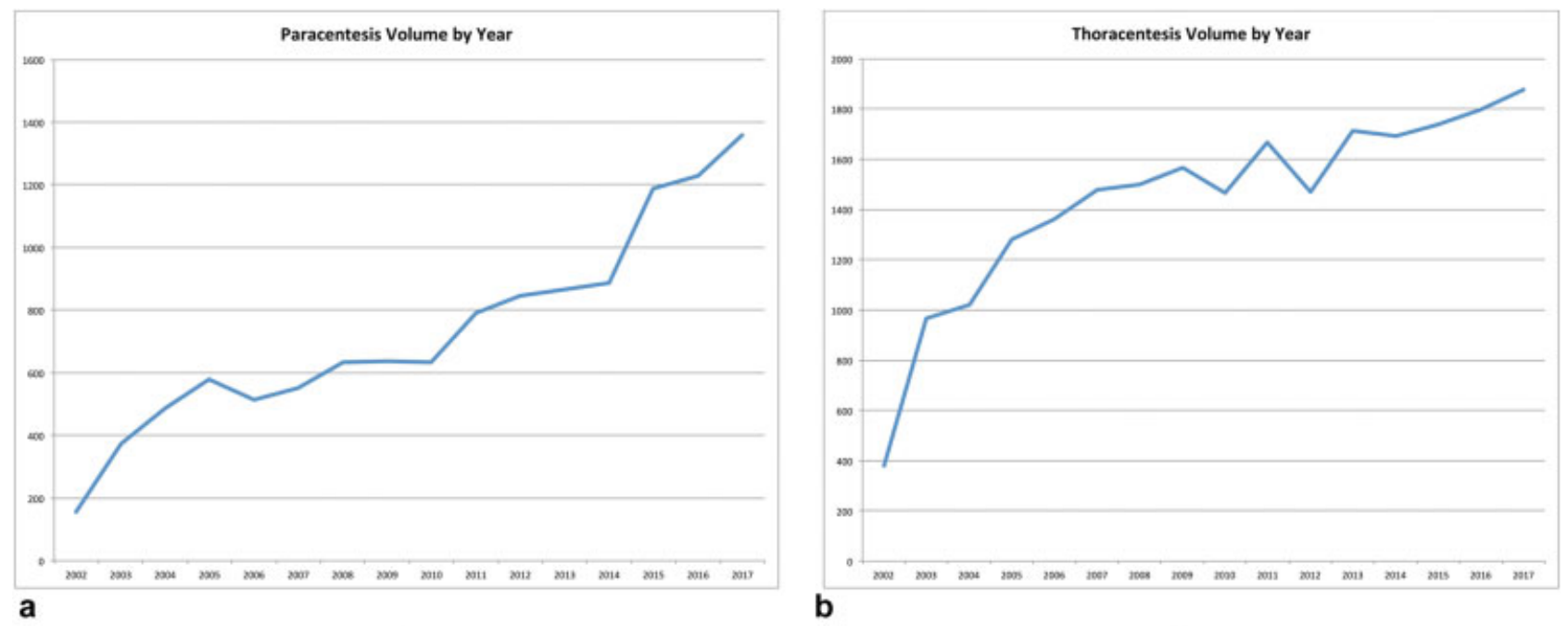

a

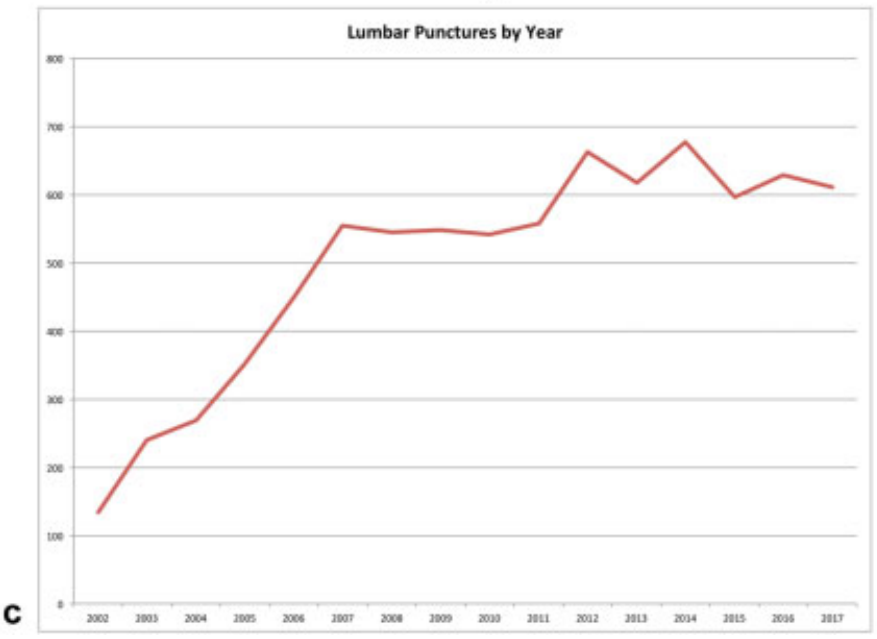

Fig. 6 Paracentesis (a), thoracentesis (b), and lumbar puncture (c) volumes by year.

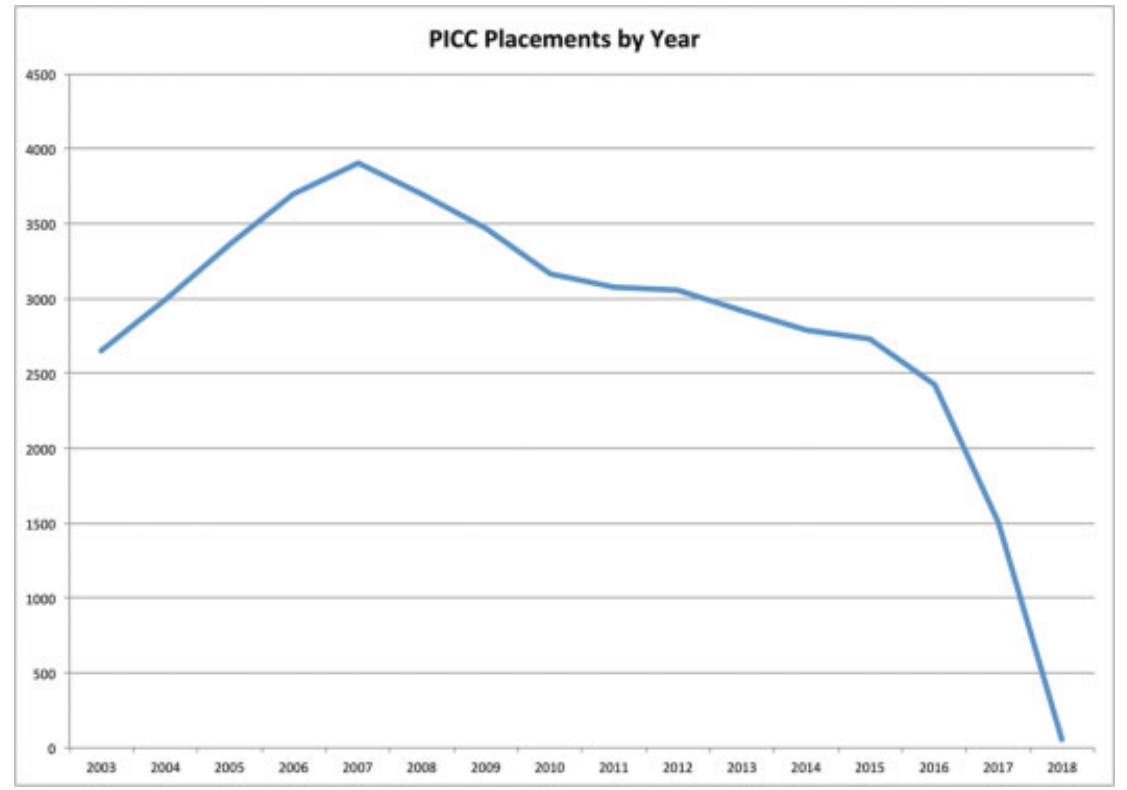

Fig. 7 Peripherally inserted central catheter (PICC) placements by year. 
minor procedures used to be performed bedside "blind," but have moved toward image-guided intervention for both patient's and referring provider's satisfaction. Examples of smaller cases include paracentesis, thoracentesis, and lumbar puncture. This increase in volume continues the trend observed by Duszak et al on Medicare beneficiary data that radiologists are now the primary providers for patients undergoing paracentesis. In contrast, our thoracentesis volumes have risen in parallel rather than declined. Overall, our experience supports their conclusions that the increase in minor procedures is "likely attributable to both the incremental safety of imaging guidance and also the unfavorable economics of these procedures."7 This increase in volume has been partially handled through utilization of advanced practice professionals (APPs) to offload the burden on IR physicians. However, the clinical rounding duties of the APPs and staffing constraints spread between multiple sites means that the majority of cases still fall to the attending physician. Work toward optimizing the utilization of nonphysicians for small procedures is difficult with many scheduling constraints, but remains an ongoing process.

PICC insertion is one smaller procedure where the data show a steady decline over the study period. This may be partially a result of national trends for reducing bloodstream infections by reducing or avoiding these types of devices. More recently, the hospital administration pushed for the formation of a vascular access service using midlevel providers separate from the IR service to provide bedside PICC placement. With the initiation of this "Vascular Access Team" in September 2017, the mean monthly PICC placement procedures dropped from 183 in the 6 months preceding the change to 8 in the 6 months after.

Despite these gains and losses in service lines over time, the overall trend is toward continued growth of procedural volumes in our IR practice. As practice patterns change, new procedures and service lines have filled the gaps left by procedures that have either gone out of favor or where referral patterns have moved to other specialties. Peripheral arterial intervention and NIR procedures represent two of our largest service lines to suffer declines related to external pressures from both hospital administration during contract negotiations and competition from other specialties.

Strategies for future success include hiring of new partners with skill sets acquired in training that add to existing or form the basis for new service lines. A dedicated IR clinic with efficient patient management to move patients through consultation, workup, procedures, and postprocedure follow-up has also been indispensable to our success. Fostering relationships with referring providers and participating in multidisciplinary conferences are key to building the referral patterns to sustain growth. Anticipating service areas with specialty overlap where an established IR presence could provide a barrier to entry to competing specialties may prevent loss of a service line, but ultimately external forces may have the final say. Finally, the recognition of IR as a distinct medical subspecialty with new training pathways focused on the clinical model of care delivery is continuing the separation of interventional and diagnostic radiologists. This may lead to a shift in IR practice models which will alter the dynamics of contract negotiations and may ultimately provide a fertile environment to regain lost ground and grow even further.

Limitations of the study are primarily a result of limitations in the data. Billing data are only available beginning in 2013. Procedural "orderable" data are only as good as the specificity of the individual codes. For example, for TACE procedures performed prior to 2014, the true volume data are unavailable because neither the billing nor orderable datasets capture the true volume of procedures. Extensive chart review could solve this limitation. Service line selection for analysis in this study was driven in part by procedural and billing data that were complete and unambiguous to minimize effects of the dataset limitations.

\section{Conclusions}

The breadth of interventional procedures performed within a particular practice is determined by the local environment. This includes a variety of internal and external forces such as referral base, administrative control, partner interest, and contract negotiations. As a large combined interventional and diagnostic radiology practice, advanced radiology services. As a large combined interventional and diagnostic radiology practice, Advanced Radiology Services, PC has experienced all of the aforementioned forces which have contributed to our successes and failures in service lines over the past 15 years. Inevitably, as minimally invasive procedures gain favor, establishing a direct referral pattern and providing beneficial and efficient outcomes are key to growing and maintaining new service lines. Some external forces are beyond avoiding, but impact to a practice can be mitigated in part by the willingness of IRs to innovate and adopt new treatments to fill care gaps in the local environment.

\section{References}

1 Murphy TP, Soares GM. The evolution of interventional radiology. Semin Intervent Radiol 2005;22(01):6-9

2 Sunshine JH, Lewis RS, Bhargavan M. A portrait of interventional radiologists in the United States. AJR Am J Roentgenol 2005;185 (05):1103-1112

3 Kallmes DF, Comstock BA, Heagerty PJ, et al. A randomized trial of vertebroplasty for osteoporotic spinal fractures. N Engl J Med 2009;361(06):569-579

4 Buchbinder R, Osborne RH, Ebeling PR, et al. A randomized trial of vertebroplasty for painful osteoporotic vertebral fractures. N Engl J Med 2009;361(06):557-568

5 Siskin G. Outpatient care of the interventional radiology patient. Semin Intervent Radiol 2006;23(04):337-345

6 Drucker EA, Brennan TA. The turf war over peripheral vascular intervention. Part I. Setting the stage. Radiology 1994;193(02): $81 \mathrm{~A}-86 \mathrm{~A}$

7 Duszak R Jr, Chatterjee AR, Schneider DA. National fluid shifts: fifteen-year trends in paracentesis and thoracentesis procedures. J Am Coll Radiol 2010;7(11):859-864 